Results: $20 / 22$ (91\%) eligible subjects enrolled in the OLE, following a mean interval of 31 weeks from the end of Part A, during which they continued to receive standard-of care treatments, to the start of the OLE during which lenabasum $20 \mathrm{mg}$ BID was added. 17/20 (85\%) subjects were on stable baseline immunosuppressive drugs. At the time of this data cut-off, 17 subjects were still enrolled, 17 had completed 140 months (2.7 years), and 15 had completed 156 months (3 years) of OLE dosing. All OLE subjects experienced at least 1 adverse event (AE), with 118 AEs during the OLE through Dec 2020. Most AEs were mild ( $n=111,94 \%$ ), with 2 severe AEs (fatigue and metastatic prostate cancer) considered unrelated to lenabasum. AEs occurring in $\geq 3 / 20$ OLE subjects were: URI $(n=5)$; fatigue $(n=4)$; nausea $(n=3)$; common cold $(n=3)$; UTI $(n=3)$; and DM flare $(n=3)$. No serious AEs related to lenabasum have been reported in this OLE to date. No subject discontinued the OLE because of an AE related to lenabasum.

Improvement was seen in multiple physician- and patient-reported efficacy outcomes. CDASI activity score improved through the first 15 months of lenabasum treatment in the OLE and remained stable thereafter, with an improvement of $\sim 20$ points from the beginning of the study maintained from Month 15 through Year 3 in the OLE. CDASI damage score increased through the first year of the OLE, even though skin activity was decreasing, but lessened thereafter, returning after 3 years to about the same level it was at the beginning of the OLE. Other outcomes shown in Figure 1 followed the same general pattern as CDASI activity score, with improvement through the first 12-16 months of the OLE, then stability thereafter. Of note, 2 subjects had disease flares shortly after stopping lenabasum for conclusion of the OLE.

Conclusion: Lenabasum continues to have a favorable safety and tolerability profile in the OLE of the Phase 2 trial JBT101-DM-001 with no serious AEs or study discontinuations related to lenabasum. The CDASI activity score and multiple other physician and patient-reported outcomes improved and have remained stable, showing durability of improvement in these patients with refractory skin disease. Skin damage was reversible and began to improve once skin activity stabilized. The limitations of attributing this improvement to lenabasum in the setting of open-label dosing is acknowledged. These data support further testing of lenabasum for the treatment of DM, and a Phase 3 study of lenabasum in DM is ongoing.
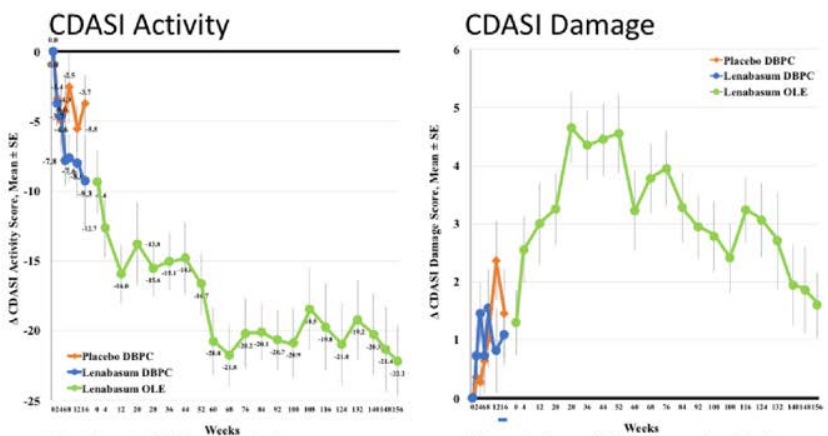

Patient Skin Activity

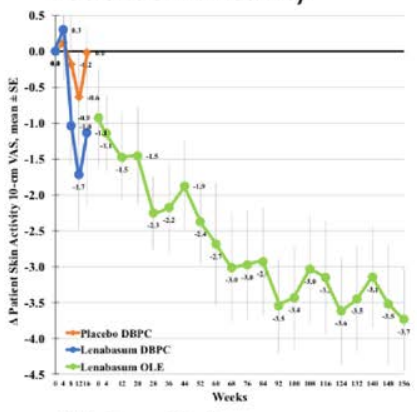

SkinDex-29 Symptoms
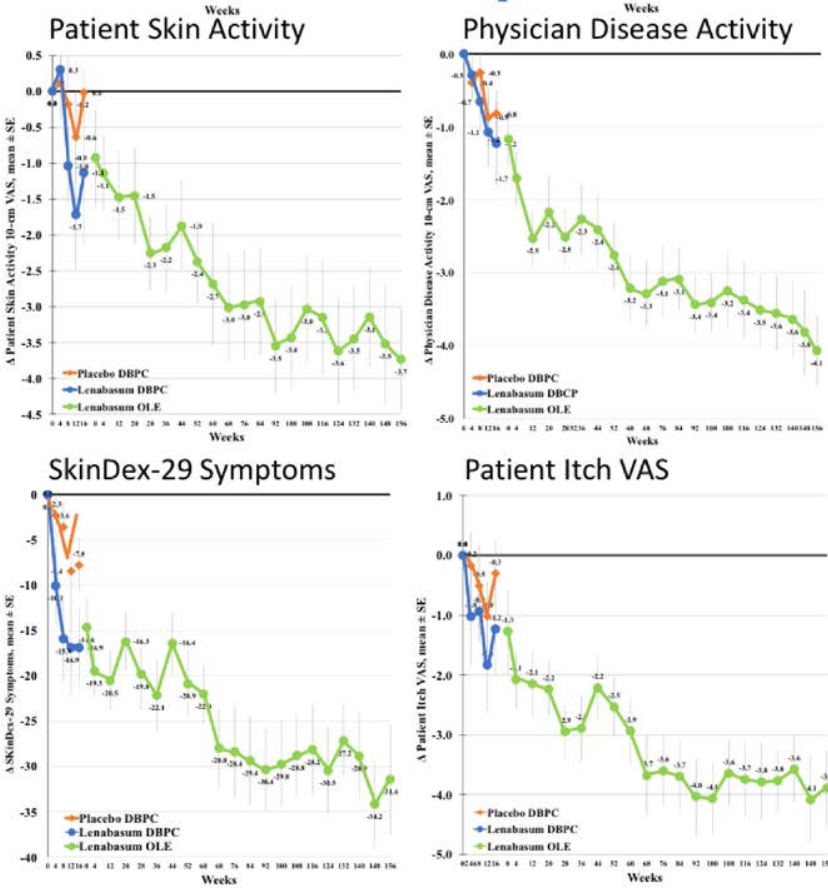

$$
\text { Patient Itch VAS }
$$

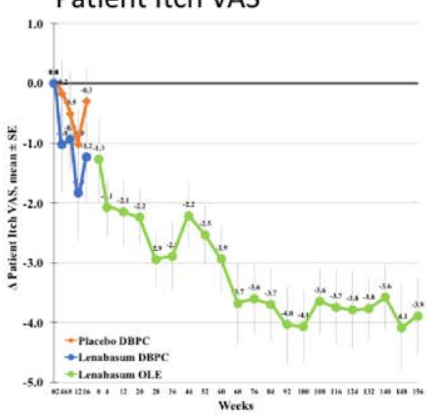

Figure 1. Change from Baseline in Selected Efficacy Outcomes in OLE of Phase 2 Trial JBT101-DM-001
Disclosure of Interests: Victoria Werth Grant/research support from: Investigator for Corbus Pharmaceuticals and received funding to conduct trials, Josef Concha: None declared, Julie Burroughs: None declared, Joyce Okawa: None declared, Rui Feng: None declared, Anisha Jobanputra: None declared, Robert Borucki: None declared, Kathleen Hally Employee of: Employee of Corbus Pharmaceuticals, Emily Hejazi: None declared, Michael Tillinger Employee of: Employee of Corbus Pharmaceuticals, Scott Constantine Employee of Employee of Corbus Pharmaceuticals, Nancy Dgetluck Employee of: Employee of Corbus Pharmaceuticals, Barbara White Employee of: Employee and stock holder of Corbus Pharmaceuticals

DOI: 10.1136/annrheumdis-2021-eular.2048

\section{POS0316 MODELLING SHORT-TERM FVC CHANGES FROM SENSCIS TO LONG-TERM FVC COURSE IN SSC- ILD DEMONSTRATES CLINICALLY MEANINGFUL REDUCTION OF FVC DECLINE AND SURVIVAL BENEFITS}

A. M. Hoffmann-Vold ${ }^{1}$, D. Huscher ${ }^{2}$, P. Airò ${ }^{3}$, E. Zanatta ${ }^{4}$, P. Carreira ${ }^{5}$, Y. Allanore ${ }^{6}$, U. Müller-Ladner ${ }^{7}$, A. Giollo ${ }^{8}$, M. R. Pozzi ${ }^{9}$, C. Souza Muller ${ }^{10}$,

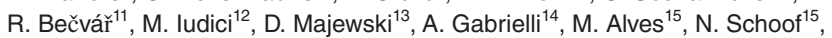
O. Distler ${ }^{16} .{ }^{1}$ Oslo University Hospital, Deptartment of Rheumatology, Oslo, Norway; ${ }^{2}$ Charité-Universitaetsmedizin, Institute of Biometry and Clinical Epidemiology, and Berlin Institute of Health, Berlin, Germany; ${ }^{3}$ ASST Spedali Civili of Brescia, University of Brescia, Rheumatology and Clinical Immunology, Brescia, Italy; ${ }^{4}$ University of Padova, Rheumatology Unit, Department of Medicine, Padova, Italy; ${ }^{5} 12$ de Octubre University Hospital, Department of Rheumatology, Madrid, Spain; ${ }^{6}$ Cochin Hospital, Department of Rheumatology, Paris, France; ${ }^{7}$ Justus Liebig University Giessen, Campus Kerckhoff, Department of Rheumatology and Clinical Immunology, Bad Nauheim, Germany; ${ }^{8}$ University of Verona, Rheumatology Section, Department of Medicine, Verona, Italy; ${ }^{9}$ University of Milan-Bicocca, Respiratory Unit, San Gerardo Hospital, Department of Medicine and Surgery, Monza, Italy; ${ }^{10}$ Universidade Federal do Parana, Division of Rheumatology, Department of Medicine, Curitiba, Brazil; ${ }^{11}$ Charles University, Department of Rheumatology, Praha, Czech Republic; ${ }^{12}$ Geneva University Hospitals and University of Geneva, Rheumatology Unit, Geneva, Switzerland; ${ }^{13}$ Poznan University of Medical Sciences, Department of Rheumatology, Rehabilitation and Internal Medicine, Poznan, Poland; ${ }^{14}$ Università Politecnica delle Marche, Dipartimento di Scienze Climiche e Molecolari, Ancona, Italy; ${ }^{15}$ Boehringer Ingelheim International GmbH, TA Inflammation Med, Ingelheim am Rhein, Germany;

${ }^{16}$ University Hospital Zurich, Department of Rheumatology, Zurich, Switzerland

Background: Nintedanib has shown to slow FVC decline by $41 \mathrm{ml}$ over 52 weeks in systemic sclerosis-associated interstitial lung disease (SSC-ILD). However, the long-term effect of nintedanib treatment on ILD progression and mortality in SSc patients is so far unknown.

Objectives: Here, the 52-week treatment efficacy of nintedanib was modeled and extrapolated on the long-term FVC course and survival in SSc-ILD patients from the European Scleroderma Trial and Research (EUSTAR) database.

Methods: SSc patients from the EUSTAR database fulfilling the inclusion criteria of the SENSCIS trial (SSc classification criteria, ILD confirmed by imaging, disease duration of $<7$ years, $F V C \geq 40 \%$ pred, diffusion capacity of the lung for carbon monoxide (DLCO) 30-89\%pred); and matched for baseline characteristics as well as matched for the 52-week FVC change of SENSCIS patients were included $(n=236)$. Linear mixed models including time, risk factors at baseline (sex, age, dyspnea class, DLCO\%, CRP, modified Rodnan skin score, SSc subtype, SSc auto-antibodies, disease duration, synovitis and muscle weakness) were used to estimate the natural FVC course over time. On this natural course of FVC, the observed effect from the SENSCIS trial representing the absolute 52-week difference of FVC decline between the nintedanib and the placebo group was applied as continuous annual effect (SENSCIS effect model). Survival was estimated for both the natural course as well as the SENSCIS effect model using Cox regression.

Results: Of the 236 included patients, $75 \%$ were females, $65 \%$ had diffuse cutaneous SSc. Mean age was 50.6 years, mean FVC $78.2 \%$ pred and DLCO $56.3 \%$ pred at time of inclusion. Mean FVC change after $12 \pm 3$ months was -2.3 $\pm 6.9 \%$ pred. These parameters were largely similar to the characteristics of the SENSCIS population.

In the longitudinal follow up of this population, the natural course of FVC showed a total FVC decline of $-16.3 \%$ pred over 5 years. With assumed SENSCIS effects (effects of nintedanib treatment reported in SENSCIS), the 5-year FVC decline was reduced to $-10.3 \%$ pred (Figure 1 ).

The reduced FVC progression translated into an improved survival. The natural 5 -year survival of this SSc-ILD population was $88.2 \%$. When extrapolating also a severe FVC decline early in the course, frequently terminated by early mortality of SSc patients excluding them from long-term outcome assessment, the estimated 5 -year survival was reduced to $81.6 \%$. When the SENSCIS effects on 
FVC were considered, the 5-year extrapolated survival was increased to $86.3 \%$ (Figure 2).

Conclusion: Long-term experience of nintedanib treatment in SSc-ILD patients is lacking so far, therefore we modeled and extrapolated the 52-week treatment efficacy of nintedanib on the long-term FVC course and survival in SSc-ILD patients from the EUSTAR database. We could demonstrate a significant reduction of FVC decline by extrapolating the annual treatment effects of nintedanib from the SENSCIS trial from 1 to 5 years in EUSTAR. Translating these reductions of FVC decline into survival, the 5-year mortality rate was reduced from $18 \%$ to $13 \%$
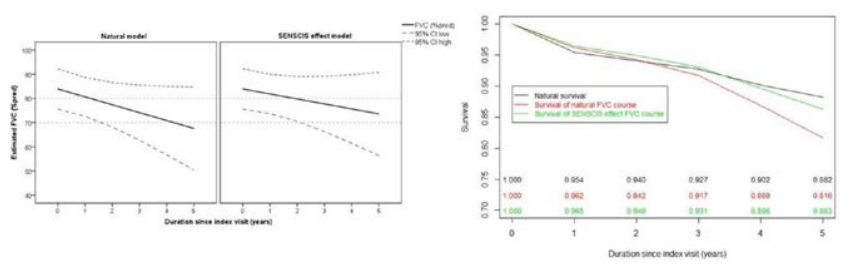

Disclosure of Interests: Anna-Maria Hoffmann-Vold Speakers bureau: Boehringer Ingelheim, Actelion, Roche, Merck Sharp \& Dohme, Lilly, Consultant of: Actelion, Boehringer Ingelheim, Roche, Bayer, Merck Sharp \& Dohme, ARXX, Lilly and Medscape, Grant/research support from: Boehringer Ingelheim, Dörte Huscher: None declared, Paolo Airò Speakers bureau: Boehringer Ingelheim, Consultant of: Bristol-Myers-Squibb, Novartis, Elisabetta Zanatta Speakers bureau: Boehringer Ingelheim, Actelion, GSK, Paid instructor for: GSK, Consultant of: Boehringer Ingelheim, GSK, Patricia Carreira Speakers bureau: Actelion, Boehringer Ingellheim, Janssen, GSK, Paid instructor for: Boehringer Ingelheim, Consultant of: AbbVie, Boehringer Ingelheim, VivaCell, Emerald Health Pharmaceuticals, Gesynta Pharma, Sanofi Genzyme, Grant/research support from: Roche, GSK, Yannick Allanore Consultant of: Honorarium received from Boehringer, Medsenic

Sanofi, Menarini, Grant/research support from: Grants received from Alpine, Ose Immunogenetics, Ulf Müller-Ladner Speakers bureau: Boehringer Ingelheim, Consultant of: Boehringer Ingelheim, Alessandro Giollo: None declared, Maria Rosa Pozzi: None declared, CAROLINA SOUZA MULLER Speakers bureau: Boehinger Ingelheim, Janssen, Roche, LIBBS, Bristol-Myers-Squib, Radim Bečvář Consultant of: Actelion, Boehringer Ingelheim, Michele ludici: None declared, Dominik Majewski Speakers bureau: Boehringer Ingelheim - 2 x paid as a speaker, Armando Gabrielli Grant/research support from: Pfizer, CSL Behring, Margarida Alves Employee of: Boehringer Ingelheim, Nils Schoof Employee of: Boehringer Ingelheim International $\mathrm{GmbH}$, Oliver Distler Speakers bureau: Boehringer Ingelheim, Medscape, IQone, Roche, Consultant of: Abbvie, Acceleron Pharma, Amgen, AnaMar, Arxx Therapeutics, Baecon Discovery, Blade Therapeutics, Bayer, Boehringer Ingelheim, ChemomAb, Corbus Pharmaceuticals, CSL Behring, Galapagos NV, Glenmark Pharmaceuticals, GSK, Horizon (Curzion) Pharmaceuticals, Inventiva, iQvia, Italfarmaco, Kymera Therapeutics, Lilly, Medac, Mitsubishi Tanabe Pharma, MSD, Novartis, Pfizer, Roche, Sanofi, Serodapharm, Topadur, Target Bioscience and UCB, Grant/research support from: Kymera Therapeutics, Mitsubishi Tanabe

DOI: 10.1136/annrheumdis-2021-eular.2522

\section{POS0317 THE PERFORMANCE OF DIFFUSING CAPACITY FOR MONOXIDE CARBON (DLCO) AND FORCED VITAL CAPACITY (FVC) IN PREDICTING THE ONSET OF SYSTEMIC SCLEROSIS (SSC)-INTERSTITIAL LUNG DISEASE (ILD) IN THE EUROPEAN SCLERODERMA TRIALS AND RESEARCH (EUSTAR) DATABASE}

G. Lepri ${ }^{1}$, C. Bruni ${ }^{1}$, L. Tofani ${ }^{1}$, A. Moggi Pignone ${ }^{2}$, M. Orlandi ${ }^{1}$, T. Sara ${ }^{3}$, M. Hughes ${ }^{4}$, F. Del Galdo ${ }^{5}$, R. Irace ${ }^{6}$, O. Distler ${ }^{7}$, V. Riccieri ${ }^{8}$, Y. Allanore ${ }^{9}, A$ M. Gheorghiu ${ }^{10}$, E. Siegert ${ }^{11}$, J. De Vries-Bouwstra ${ }^{12}$, E. Hachulla ${ }^{13}$, M. Tikly ${ }^{14}$, N. Damjanov ${ }^{15}$, F. Spertini ${ }^{16}$, L. Mouthon ${ }^{17}$, A. M. Hoffmann-Vold ${ }^{18}$, A. Gabrielli ${ }^{19}$ S. Guiducci ${ }^{1}$, M. Matucci-Cerinic ${ }^{1}$, D. Furst ${ }^{1,20}$, S. Bellando Randone ${ }^{1}$ on behalf of EUSTAR. ${ }^{1} \mathrm{AOU}$ Careggi, University of Florence, Division of Rheumatology, Florence, Italy; ${ }^{2} \mathrm{AOU}$ Careggi, University of Florence, Division of Internal Medicine, Florence, Italy; ${ }^{3} \mathrm{AOU}$ Careggi, University of Florence, Interventional Pulmonology Unit, Florence, Italy; ${ }^{4}$ Royal Hallamshire Hospital, Sheffield Teaching Hospitals NHS Foundation Trust, Department of Rheumatology, Sheffield, United Kingdom; ${ }^{5}$ NIHR Biomedical Research Centre and Leeds Institute of Rheumatic and Musculoskeletal Medicine, University of Leeds, Raynaud's and Scleroderma Programme, Leeds, United Kingdom; ${ }^{6}$ University of Campania "Luigi Vanvitelli", Rheumatology Unit, Department of Precision Medicine, Naples, Italy; ${ }^{7}$ Univ Hospital Zurich, University of Zurich, Department of Rheumatology, Zurich, Switzerland; ${ }^{8}$ Rheumatology Unit, "Sapienza" University, Rome, Italy; ${ }^{9}$ Hopital Cochin, University of Paris, Rheumatology
Department, Paris, France; ${ }^{10}$ Cantacuzino Hospital, Carol Davila University of Medicine and Pharmacy, Internal Medicine \& Rheumatology Department, Bucharest, Romania; ${ }^{11}$ Charite University Hospital, Rheumatology, Berlin, Germany; ${ }^{12}$ Leiden University Medical Center, Department of Rheumatology, Leiden, Netherlands: ${ }^{13}$ Centre Hospitalier Universitaire, Service de Médecine Interne, Lille, France; ${ }^{14}$ Chris Hani Baragwanath Academic Hospital University of the Witwatersrand, Division of Rheumatology, Department of Internal Medicine, Johannesburg, South Africa; ${ }^{15}$ University Belgrade Medical School, Institute of Rheumatology, Belgrade, Serbia; ${ }^{16} \mathrm{CHUV}, 16$. Service Immunologie et Allergie, Lausanne, Switzerland; ${ }^{17}$ National Referral Center for Rare Systemic Autoimmune Diseases, Hôpital Cochin, University Paris Descartes, Paris, France; ${ }^{18}$ Oslo University Hospital, Department of Rheumatology, Oslo, Norway; ${ }^{19}$ Università Politecninca delle Marche, Department of Clinical and Molecular Science, Ancona, Italy; ${ }^{20}$ University Califonia Los Angeles, Division Rheumatology, Los Angeles, United States of America

Background: In SSc, ILD is a major cause of morbidity and mortality. High resolution computed tomography (HRCT) is the gold standard for the diagnosis Predictors of ILD onset are eagerly awaited to improve SSc-ILD management Pulmonary function test (PFTs) are routinely performed to measure lung function changes.

Objectives: Our aim was to investigate the performance of DLCO (diffusing capacity of lung carbon monoxide) and FVC (forced vital capacity) in predicting the development of SSc-ILD.

Methods: The longitudinal data of DLCO, FVC and ILD on HRCT of SSc patients from the EUSTAR database were evaluated at baseline $\left(t_{0}\right)$, after $12( \pm 4)\left(t_{1}\right)$ and $24( \pm 4)\left(t_{2}\right)$ months. Patients with negative HRCT for any sign of ILD both at $t_{0}$ and $t_{1}$ were included. Patients who presented or developed pulmonary hypertension during the study period were excluded. At baseline, demographic data, disease duration from Raynaud's onset, disease subsets, autoantibodies and other laboratory and instrumental data were recorded.

Results: $474 / 17805$ patients were eligible for the study (403 females, 71 males): $26.0 \%$ dcSSc, $58.3 \%$ IcSSc, $220(48.0 \%)$ patients with positive anticentromere antibodies (ACA) and $117(25.4 \%)$ with positive antitopoisomerase I antibodies (Topo-I abs). Among all enrolled patients, 46 (9.7\%) developed HRCT signs of ILD at $t$. Patients with Topo-I abs showed an association with ILD development at $\mathrm{t}_{2}(16.7 \%$ vs $7.8 \%, \mathrm{p}=0.0031)$, contrarily ACA positive patients were negatively associated with ILD appearance after 2 years of follow-up (4.4\% vs $14.4 \%$, $\mathrm{p}=0.0001$ ). Positive $\mathrm{t}_{2}$ HRCT patients had a significant lower value of DLCO and FVC at all three assessments when compared to patients with a negative HRCT at $t_{2}$ (Table 1) and both $t_{0}$ DLCO and FVC values negatively correlated with ILD development (Table 1). The mean $t_{\text {to }} t_{1}$ change $(\Delta)$ of DLCO in patients with negative $t_{2}$ HRTC and positive $t_{2}$ HRCT were $-0.5( \pm 12.6)$ and $-1.0( \pm 15.1)$, respectively. The mean $t_{0}$ to $t_{1} \Delta F V C$ in patients with negative $t_{2} H R T C$ and pos itive $t$ HRCT were $-0.2( \pm 10.6)$ and $0.1( \pm 11.5)$, respectively. None of them predicted the appearance of ILD at $t_{2}$ ( $\triangle D L C O:$ OR (IC) 0.997 (0.97-1.02), $p=0.8024$ $\triangle$ FVC OR (IC) $1.002(0.97-1.03), p=0.8664)$. The data showed an association between $t_{0}$ DLCO value $<80 \%$ and ILD appearance after 2 years of follow-up [OR(IC): 3.09(1.49-6.40), $p=0.0023]$. Such association was not observed for $t$ FVC value $<80 \%$ [OR(IC): $1.95(0.81-4.68), p=0.1329]$. The predictive capability of t $\mathrm{DLCO}<80 \%$ was moderate but stronger than FVC $<80 \%$ [AU ROC: $0.62(0.56$ $0.69), 0.53(0.48-0.59)$ respectively, $\mathrm{p}=0.0205]$.

Conclusion: Our data suggest that an impaired baseline DLCO $(<80 \%)$ may have a predictive value for the development of ILD on HRCT after 2 years of follow-up. Further rigorous prospective studies are warranted to understand the role of DLCO evaluation in the course of SSc.

Table 1. DLCO and FVC values at $t_{0}, t_{1}$ and $t_{2}$ values in patients with positive or negative HRCT for ILD at $t_{2}$ and their statistical differences.

\begin{tabular}{|c|c|c|c|c|}
\hline & $\begin{array}{l}\text { Patients without ILD } \\
\text { t2 }(\text { mean } \pm S D)\end{array}$ & $\begin{array}{r}\text { ents with ILD } \\
(\text { mean } \pm \text { SD) }\end{array}$ & OR (95\%CL) & $\mathrm{p}$-value \\
\hline DLCO at $t_{0}$ & $79.0 \pm 16.6$ & $69.9 \pm 17.4$ & $0.97(0.95-0.99)$ & 0.0006 \\
\hline DLCO at $t^{\circ}$ & $78.4 \pm 16.8$ & $68.9 \pm 18.6$ & $0.97(0.95-0.98)$ & 0.0005 \\
\hline DLCO at $t^{1}$ & $78.0 \pm 17.0$ & $65.1 \pm 19.1$ & $0.95(0.93-0.97)$ & $<0.0001$ \\
\hline FVC at $t^{2}$ & $102.2 \pm 17.3$ & $94.6 \pm 16.2$ & $0.97(0.96-0.99)$ & 0.0052 \\
\hline FVC at $t^{0}$ & $101.9 \pm 17.9$ & $94.7 \pm 16.5$ & $0.98(0.96-0.99)$ & 0.0092 \\
\hline FVC at $t_{2}$ & $101.6 \pm 17.6$ & $94.5 \pm 20.0$ & $0.98(0.96-1)$ & 0.0126 \\
\hline
\end{tabular}

Disclosure of Interests: Gemma Lepri: None declared, Cosimo Bruni Speakers bureau: $\mathrm{CB}$ reports personal fees from Actelion, personal fees from Eli Lilly, Grant/ research support from: $C B$ reports personal fees from Actelion, personal fees from Eli Lilly, grants from European Scleroderma Trial and Research (EUSTAR) group, grants from New Horizon Fellowship, grants from Foundation for Research in Rheumatology (FOREUM), grants from Fondazione Italiana per la Ricerca sull'Artrite (FIRA), outside the submitted work, Lorenzo Tofani: None declared, Alberto Moggi Pignone: None declared, Martina Orlandi: None declared, Tomasetti Sara Speakers bureau: Speaker's fees for Roche and Boehringer Ingelheim 